\section{DSM-5. ¿La incorporación definitiva de la psiquiatría en la medicina?}

\author{
GUSTAVO FIGUEROA ${ }^{1}$
}

\section{DSM-5: the definitive inclusion of psychiatry in medicine?}

DSM-5 is a significant factor in promoting the "remedicalization" of psychiatry as the focus of psychiatric knowledge, developed by the evidence-based medicine movement, shifted from the clinically-based biopsychosocial model to a research-based medical model. DSM-5 purposes are 1]clinical: diagnosis, prevention, early identification, management, outcome, assessment of improvement; 2] clinical research: etiology, course, effective treatments, cost-effective treatments, reliability and validity and utility of diagnosis; 3] a worldwide common language of diagnostic criteria used by mental health professionals; and 4] to improve communication with users of services, caregivers, and society in general. In the absence of a "gold standard" there are two basic questions still without answers 1] what kind of entities are psychiatric disorders?; and 2] How to integrate the multiple explanatory perspectives of psychiatric illness?.

(Rev Med Chile 2019; 147: 475-479)

Key words: Diagnostic and Statistical Manual of Mental Disorders; Mental Disorders; Validation Studies.
'Departamento de Psiquiatría,

Universidad de Valparaíso.

Valparaíso, Chile.

Correspondencia a:

Dr. Gustavo Figueroa Departamento de Psiquiatría, Universidad de Valparaíso.

Avenida Leopoldo Carvallo 200.

Valparaíso, Chile.

gfigueroacave@gmail.com
$\mathrm{N}$ acida a comienzos del siglo XIX ${ }^{1}$, la psiquiatría ha buscado incorporarse a la medicina fundada científicamente adoptando sus principios, metodología y procedimientos ${ }^{2}$. Empero su índole epistemológica híbrida -ciencia natural, ciencia humana- ${ }^{3}$, ha condicionado poner en cuestión repetidamente su estatuto científico esencial, influido por factores ideológicos, sociales, económicos y políticos ${ }^{4}$. El DSM-5, "Manual diagnóstico y estadístico de los trastornos mentales" (2013), representa la culminación de cuatro décadas de investigación por incorporarse al movimiento de ciencia médica basada en la evidencia ${ }^{5,6}$. Este trabajo procura indagar si el DSM-5 ha conseguido finalmente que la psiquiatría supere su condición de práctica empírica que la sociedad le ha asignado tradicionalmente ${ }^{7}$. Se considerarán antecedentes, fundamentos, concepto de trastorno mental, evidencias y situación actual.

\section{Antecedentes}

En la década del 60 tres factores incidieron en la psiquiatría: rechazo al diagnóstico por su connotación de etiqueta injuriosa ${ }^{8}$, imposibilidad para comparar los diagnósticos entre diferentes clínicos por ausencia de una terminología unitaria ${ }^{9} y$ limitaciones para evaluar la eficacia de las terapias farmacológicas recién introducidas por carencia de criterios estandarizados ${ }^{10}$. Entre 1970 y 1978 un equipo de la Universidad de Washington formuló un modelo médico diferente del biopsicosocial tradicional de la psiquiatría, desarrolló criterios diagnósticos operacionales (RDC), reintrodujo la importancia del curso y desenlace de las afecciones y determinó que los diagnósticos debían basarse en evidencia empírica ${ }^{11,12}$. Nació el DSM-III (1980), que consistió en pasar de las manifestaciones sintomatológicas a la fiabilidad, de la descripción a la evidencia, de la utilidad a la validez, del clínico 
al investigador ${ }^{13}$, condiciones que continuaron desarrollándose en manuales ulteriores ${ }^{14}$. Empero surgieron dificultades básicas: alta comorbilidad, aumento excesivo de casos "no especificados de otra manera" y estudios epidemiológicos ineficaces para diferenciar los síndromes diagnósticos; esto es, aunque se postuló la existencia de entidades definidas en la clínica, éstas se probaron como altamente heterogéneas, imprecisas y condicionadas por la estructura politética de la clasificación con niveles de corte arbitrarios antes que por datos empíricos ${ }^{15}$.

\section{Fundamentos}

Heidegger ha señalado que la medicina, en cuanto ciencia, "le atañe solamente a lo que su modo de concebir ha admitido previamente como posible objeto para ella"16; movido por el proyecto-previo (Vor-entwurf) del investigador, este im-pone y se queda sólo con "lo escogido", vale decir, lo "enfermo" y, dentro de este, trata de sustituirlo por otro más riguroso, la precisión objetiva. "La certeza" que se alcanza es producto del dominar, instrumentalizar, clasificar ${ }^{17,18}$, o sea, ser operacional es forzoso porque es obra del im-poner que ob-liga a quedarse sólo con "lo escogido", lo ob-jetivado, ya que coloca delante el asunto que estudia en tanto objeto ${ }^{19}$.El DSM-5 procede de igual manera y sigue las indicaciones de Carnap de atenerse a "los datos de hecho", emitir "enunciados observacionales", llevar a cabo "verificaciones empíricas" y traducirlas en un "lenguaje exacto"20. Esto es, examina la variada relación entre los componentes biológicos, semánticos y sociales $\mathrm{y}$, en un proceso progresivo de calibración, va comparando la correspondencia entre el lenguaje de su descripción con el objeto de su investigación ${ }^{21}$.

Concepto de trastorno mental. La medicina es un saber técnico, apunta Heidegger, que utiliza un pensar calculador (rechnend) que des-oculta haciendo aparecer los entes como mensurables, como ob-jetos del mundo operables con números, y así la enfermedad se convierte en positiva: es un "hecho" calculable ${ }^{22}$. El DSM-5 lo respetó, precisando que todo hecho positivo necesita ser verificado o falseado en estudios rigurosos expresados en cifras $^{23}$. En la etapa de elaboración el equipo del DSM-5 adoptó el concepto bivalente o híbrido de trastorno mental-disfunción dañina, prescindiendo de las concepciones escéptica antipsiquiátrica, valorativa, desviación estadística, prototipo, desventaja biológica y operacional (malestar o incapacidad $)^{24,25}$. Empero priorizó lo dimensional, que fundamenta los trastornos mentales en pares contrapuestos: objetivista y evaluativa, causal y descriptiva, esencialista y nominalista, internalizada y externalizada, entidad y agente, categorial y dimensional ${ }^{26}$. Al caracterizarlo finalmente como síndrome o patrón clínicamente significativo que refleja una disfunción en los procesos biológicos y que se acompaña de malestar o incapacidad en las actividades sociales y ocupacionales, determinó valerse, en diferente proporción, de modelos prácticos: orgánico médico, nominalista, dimensional, tipos prácticos, interpersonal y narrativo ${ }^{27,28}$. Su decisión de no comprometerse con un modelo único revela el carácter ambiguo, impreciso del concepto de trastorno en psiquiatría, enfatizando que la decisión requiere ser reevaluada empíricamente en el futuro. Sin embargo, 1] su especificación como alteraciones de la regulación emocionales, cognitivas y conductuales; 2 ] su diferenciación de las respuestas esperables y aprobadas al interior de diferentes ambientes culturales; 3 ] su separación de las conductas sociales desviadas, y 4] su independencia de los conflictos al interior de la sociedad, señalan que, aunque se está en el seno de la medicina, su índole es particular, propia y específica. Resumiendo, lo decisivo está en que los estudios de campo han mostrado que los límites o líneas divisorias entre los trastornos son difusos, las fronteras son equívocas, están en un territorio borroso-"zonas de rareza”-respecto a sus demarcaciones, de modo que permanece abierta la pregunta central: ¿qué tipo de entes son los trastornos psiquiátricos? ¿esencias reales? ¿conceptos socialmente construidos? ¿herramientas útiles prácticas? ¿redes de mecanismos causales? ${ }^{29,30}$.

Por otra parte, el apartado "otras condiciones que pueden ser foco de atención clínica” (ej. abuso infantil) señala que la psiquiatría aborda asuntos que además desbordan la medicina tradicional y demandan ser considerados porque pueden afectar el diagnóstico, pronóstico o terapia sin ser propiamente trastornos ${ }^{31}$. Aunque estas condiciones conllevan un triple peligro: mal uso o comprensión errónea (ej. vicio) ${ }^{32}$, intromisión indebida en ámbitos distintos como en el forense $\mathrm{e}^{33}$ y producción ilegítima de patología en la vida co- 
tidiana creando trastornos en dilemas inherentes a la existencia humana ${ }^{34}$.

El DSM-5 divide en 22 categorías su ámbito propio y considera la evolución de los trastornos durante el ciclo vital, describe los elementos comunes entre ellos siguiendo un enfoque desde-abajo(bottom-up), es asistemático, no obedece a una jerarquía diagnóstica, no considera criterios etiológicos, no utiliza hallazgos epidemiológicos, configura agrupaciones de nivel superior con bajo sustento empírico, profundiza en los fundamentos de manuales anteriores (Tabla 1).

La insuficiencia de datos proporcionados por la investigación requirió agregar un acápite sobre condiciones para futuros estudios-aunque estos

\section{Tabla 1. Criterios diagnósticos del DSM-5*}

Trastornos del desarrollo neurológico

Espectro de la esquizofrenia y otros trastornos psicóticos

Trastorno bipolar y trastornos relacionados

Trastornos depresivos

Trastornos de ansiedad

Trastorno obsesivo-compulsivo y trastornos relacionados

Trastornos relacionados con traumas y factores de estrés

Trastornos disociativos

Trastorno de síntomas somáticos y trastornos relacionados

Trastornos alimentarios y de la ingestión de alimentos

Trastornos de la excreción

Trastornos del sueño-vigilia

Disfunciones sexuales

Disforia de género

Trastornos destructivos, del control de los impulsos y de la conducta

Trastornos relacionados con sustancias y trastornos adictivos

Trastornos neurocognitivos

Trastornos de la personalidad

Trastornos parafílicos

Otros trastornos mentales

Trastornos motores inducidos por medicamentos y otros efectos adversos de los medicamentos

Otras problemas que pueden ser objeto de atención clínica

*Adaptado de: American Psychiatric Association. Diagnostic and Statistical Manual of Mental Disorders. $5^{\text {th }}$ edition. Arlington, VA. American Psychiatric Association; 2013. se encuentran ya en un período significativo de avance-,al cual se adiciona un apartado especial para los trastornos de personalidad que, al ser evaluados de manera dimensional y no categorial, fue rechazado por la comisión encargada del proyecto después de intensas discusiones, aunque acentuando la necesidad de ulteriores estudios de campo mensurativos ${ }^{35}$ (Tabla 2).

El comité de expertos intentó agrupar los trastornos en racimos (clusters) abarcadores superiores -meta-estructuras- que superaran la descripción tradicional, obedeciendo a criterios de validación empírica rigurosa, pero fracasó por dos motivos: falta de sustento positivo y una gran cantidad de trastornos no pudieron ser agrupados, aunque los racimos quedaron sujetos a pesquisas futuras $^{36}$ (Tabla 3).

\section{Evidencias}

El DSM-5 proyectó superar el sistema basado en el juicio clínico y para ello acudió a criterios de validez utilizados en medicina: los marcadores biológicos; con ello, cuadros de presentación clínicamente diferentes pueden detentar igual etiología subyacente. Pero los avances en psiquiatría todavía no han alcanzado el nivel de desarrollo como para utilizarlos y así necesitó recurrir a una serie de validadores como preparación para subsecuentes encuestas de campo. Aprovechó los validadores del DSM-III empero aumentándolos de 5 a 11 y los dividió en: 1] Validadores de factores de riesgo:

\section{Tabla 2. Condiciones para estudios ulteriores*}

Síndrome de psicosis atenuada
Episodios depresivos con hipomanía de corta duración
Trastorno por duelo persistente complejo
Trastorno por uso de cafeína
Trastorno de juego por internet
Trastorno neuroconductual asociado con exposición pre-
natal al alcohol
Trastorno suicida conductual
Auto-daño no suicida
Modelo alternativo del DSM-5 para los trastornos de
personalidad

*Adaptado de: American Psychiatric Association. Diagnostic and Statistical Manual of Mental Disorders. $5^{\text {th }}$ edition. Arlington, VA. American Psychiatric Association; 2013. 
Tabla 3. Meta-estructura para el DSM-5*

Racimo neurocognitivo
Delirium, demencias, trastornos amnésicos y otros
trastornos cognitivos
Racimo del desarrollo neuronal
Retardo mental, trastornos del aprendizaje, habilidades
motoras, comunicación y desarrollo invasivo
Racimo psicótico
Esquizofrenia y psicosis relacionadas, trastorno bipolar,
personalidad esquizotípica
Racimo emocional
Depresión unipolar, distimia, angustia generalizada,
pánico, fobias, obsesivo-compulsivo, dismorfofobia
corporal, hipocondría, estrés postraumático, trastornos
de ajuste, somatomorfos, personalidad evitadora
Racimo externalizador
Trastornos relacionado con sustancias, personalidades
antisocial y limítrofe, control de impulsos, trastorno de
la conducta
Trastornos no asignados en ningún racimo
Trastornos de eliminación y tics; alimentación, comer
y otros trastornos de la infancia y juventud; persona-
lidades paranoide, esquizoide, histriónica, narcisista,
evitadora, obsesiva-compulsiva; dismorfofobia corpo-
ral; trastornos de ajuste, facticio y disociativo; sueño
primario; trastornos sexuales e identidad de género;
trastornos alimentarios

*Adaptado de: Andrew G, Goldberg DP, Krueger WT, Carpenter WT, Hyman SE, Sachdev P, Pine DS. Exploring the feasibility of a meta-structure for DSM-V and ICD-11: could it improve utility and validity? Psychological Medicine 2009; 39: 1993-2000

genéticos, familiares, ambientales específicos compartidos, sustratos neurales compartidos, marcadores biológicos compartidos y antecedentes temperamentales compartidos; y 2] Validadores de cuadros clínicos: anormalidades compartidas de procesamientos cognitivos y emocionales, similitud de síntomas, altas tasas de comorbilidad, curso de la enfermedad y respuesta al tratamiento.

El verbo griego diagigôskein-diagnosticar-significaba tanto distinguir, discernir como conocer atravesando pero profundizando ${ }^{37}$. Así tres cuestiones señalan las insuficiencias en la base del DSM-5: cuadros clínicos demasiado alejados de los hallazgos de las neurociencias, categorías descriptivas con escasa relación con procesos fisiopatológicos y ausencia de conceptualización de los mecanismos disfuncionales esenciales subyacentes. Por ello se ha propuesto separar la clínica del marco propio para la investigación y crear, junto al DSM-5, el RDoC, Criterios de Dominio de la Investigación, éste último dedicado a los descubrimientos en genómica y neurociencias, que propondrá una clasificación producto de los hallazgos ${ }^{38}$; en resumen, se trata de progresar del juicio clínico a la evidencia científica, del "manual" a la "nosología".

\section{Situación actual}

1] El DSM-5 significa entrar definitivamente en la medicina científica actual basada en la evidencia siguiendo su metodología rigurosa; 2] Empero la psiquiatría posee características propias y poli dimensionales, por lo que precisa diversos niveles de explicación, que contrastan con el específico de la medicina: a]nivel único de explicación(fuerte, confianza causal, generalizable, especificidad, perfectible, proximidad, generatividad) y que b] fundamenta su nosología en la etiología ${ }^{39} ; 3$ ] Los niveles de explicación del DSM-5 se sustentan en mecanismos de niveles-cruzados, confusos que no corresponden a relaciones propiamente causales como en medicina sino predictivo-correlativas, por lo que comprometen e involucran diferentes áreas; 4] Frente al modelo médico estricto de la medicina, el DSM-5 se conforma con un modelo médico moderado que se basa en una teoría coherente de la verdad o realismo limitado, necesitado de una ontología "débil" donde la enfermedad no se la concibe como una estructura-real-existente-afuera ${ }^{40}$; 5] El DSM-5 es útil clínicamente, se puede aplicar en distintos ámbitos culturales, favorece la investigación, es perfectible incorporando los nuevos hallazgos empíricos, está homogeneizado terminológicamente con el futuro CIE-11 de la Organización Mundial de la Salud.

\section{Referencias}

1. Figueroa G. Historia de la psiquiatría. En Ivanovic-Zuvic F, Correa E, Florenzano R, Editores. Texto de Psiquiatría. Santiago: Ediciones de la Sociedad de Neurología, Psiquiatría y Neurocirugía; 2017. p. 46-61.

2. Kendler KS. The structure of psychiatric science. Am J Psychiatry 2014; 171: 931-8.

3. Marková IS, Berrios GE. Epistemology of psychiatry. Psychopathology 2012; 45: 220-7.

4. Karlson H, Hamppinen M. Biological psychiatry and reductionism. Empirical findings and philosophy. Brit J Psychiatry 1995; 167: 434-8. 
5. American Psychiatric Association. Diagnostic and Statistical Manual of Mental Disorders. 5th edition. Arlington, VA. American Psychiatric Association; 2013.

6. Evidence-based Medicine Working Group. Evidence-based medicine. A new approach to teaching the practice of medicine. JAMA 1992; 268: 2420-5.

7. Foucault M. Le pouvoir psychiatrique. Cours au Collège de France. 1973-1974. Paris: Gallimard-Seuil; 2003.

8. Szasz T. The mith of mental illness. New York: HarperCollins; 2010.

9. Cooper JE, Kendell RE, Gurland BJ, Sharpe L, Copeland JRM, Simon R. Psychiatric diagnosis in New York and London. New York and London: Oxford University Press; 1972.

10. Klerman GL, Vaillant GE, Spitzer RL, Michels R. A debate on DSM-III. Am J Psychiatry 1984; 141: 539-53.

11. Spitzer RL, Endicott J, Robins E. Research Diagnostic Criteria for a selected group of functional disorders. New York: New York Psychiatric Institute; 1975.

12. Spitzer RL, Endicott J, Robins E. Research Diagnostic Criteria: rationality and realability. Arch Gen Psychiatry 1978; 35: 773-82.

13. American Psychiatric Association. Diagnostic and Statistical Manual of Mental Disorders. 3rd edition. Arlington, VA. American Psychiatric Association; 1980.

14. American Psychiatric Association. Diagnostic and Statistical Manual of Mental Disorders. 4th edition . Arlington, VA. American Psychiatric Association; 1994.

15. Regier DA. Dimensional approaches to psychiatric classification: refining the research agenda for DSM-V: an introduction. Int J Methods Psychiatr Res 2007; 16: S1-S5.

16. Heidegger M. Die Überwindung der Methaphysik. Gesamtausgabe 7. Frankfurt: Klostermann; 2000. p. 38-51.

17. Heidegger M. Wissenschaft und Besinnung. Vortäge und Aufsätze. 5. Aufl. Pfullingen: Neske; 1967. p. 41-66.

18. Heidegger M. Das Ding. Gesamtausgabe 7. Frankfurt: Klostermann; 2000. p. 157-75.

19. Heidegger, M. Sein und Zeit. 10. Aufl. Tübingen: Niemeyer; 1963.

20. Carnap R. Der logische Aufbau der Welt. Hamburg: Felix Meiner; 1998.

21. Berrios GE. Hacia una nueva epistemología de la psiquiatría. Buenos Aires: Polemos; 2011.

22. Heidegger M. Die Frage nach der Technik. Gesamtausgabe 7. Frankfurt: Klostermann; 2000. p. 5-36.

23. Popper KR. The logic of scientific discovery. London: Hutchinson; 1959.

24. Wakefield JC. Evolutionary versus prototype analyses of the concept of disorder. J Abn Psychology 1999; 108: 374-99.
25. Lilienfeld SC, Marino L. Essencialism revisited: evolutionary theory and the concept of mental disorder. J Abn Psychology 1999; 108: 400-11.

26. Stein DJ, Phillips KA, Bolton D, Fulford KWW, Sadler JZ, Kendler KS. What is a mental/psychiatric disorder? From DSM-IV to DSM-V. Psychological Medicine 2010; 40: 1759-65.

27. Zachar P. The practical kinds model as a pragmatist theory of classification. Philo Psychol Psychiatr 2003; 9: 219-27.

28. First MB, Wakefield JC. Defining "mental disorder" in DSM-V. Psychological Medicine 2010; 40: 1779-82.

29. Kendler KS, Zachar P, Craver C. What kind of things are psychiatric disorders? Psychological Medicine 2011; 41: 1143-50.

30. Zachar P. Psychiatric disorders: natural kinds made by the world or practical kinds made by us? World Psychiatry 2015; 14: 288-90.

31. Kendler KS. Explanatory models for psychiatric illness. Am J Psychiatry 2008; 165: 695-702.

32. First MB. Clarifying the relationship between vice and mental disorder: vice as a manifestation of a psychological dysfunction. Philo Psychiat Psycholog 2008; 15: 35-8.

33. Foucault M. Les anormaux. Cours au Collège de France. 1974-1975. Paris: Seuil/Gallimard; 1999.

34. Wakefield J, First M. Clarifying the distinction between disorder and non-disorder: confronting the overdiagnosis ("false positives") problem in DSM-IV. En Phillips K, First M, Pincus H, Editors. Advancing DSM: Dilemmas in psychiatric diagnosis. Washington: American Psychiatric Association; 2003. p. 23-56.

35. Morey LC, Benson KT, Skodol AE. Relating DSM-5 section III personality traits to section II personality disorder diagnoses. Psychological Medicine 2016; 46: 647-55.

36. Wittchen HU, Beesdo K, Gloster AT. A new meta-structure of mental disorders: a helpful step into the future or a harmful step back to the past? Psychological Medicine 2009; 39: 2083-9.

37. Laín Entralgo P. El diagnóstico médico. Historia y teoría. Barcelona: Salvat; 1982.

38. Garvey M, Heinssen R, Pine DS, Quinn K, Sanislow C, Wang P. Research Domain Criteria (RDoC): toward a new classification framework for research on mental disorders. Am J Psychiatry 2010; 167: 748-51.

39. Kendler KS. Levels of explanation in psychiatric and substance use disorders: implications for the development of an etiologically based nosology. Molecular Psychiatry 2012; 17: 11-21.

40. Vattimo G, Rovatti PA, Editores. El pensamiento débil. Madrid: Cátedra; 2000. 